\title{
Airline Head-Up Display Systems: Human Factors Considerations
}

\author{
Ryan J Nichol*
}

Massey University, College of Business, New Zealand

\begin{abstract}
As the key source of information for pilots, the human visual system has necessarily driven much of the evolution in cockpit technology. In contrast to the complicated, gauge-based systems of the past, the electronic flight displays of today's modern airliners are testament to advances in human factors engineering. The next step in flight instrumentation, although already used for some 50 years in the military, is just beginning to emerge in civil transport aircraft. Head-up displays (HUDs) allow pilots to see key flight instrumentation while viewing the outside world. The need to look down at the flight instruments is removed by the HUD, resulting in increased situational awareness and greater precision in aircraft control. While the head-up display is a welcome development, as with many instances of human-technology interface, the benefits provided by HUDs are not without potential drawbacks. The present report provides a thorough grounding in key areas of importance to the development and operation of HUD systems, including the human visual system, the history and development of conventional cockpit instrumentation, and a summary of the technical aspects and human factors considerations relating to HUD systems. This basis is built upon with a more practical assessment of the HUD system used in a modern airliner, the Boeing 787.
\end{abstract}

Keywords: Head-up display; HUD; Airline; aviation; Human factors; Visual attention; Attention capture; Cognitive tunneling; Boeing 787

\section{Introduction}

Since the advent of powered flight, there have been continuous enhancements to aviation technology resulting in faster, more reliable and safer air travel. In parallel to the leaps and bounds achieved in mechanical engineering, there has been increasing understanding of the physiological and psychological limitations of human beings, and how these can best be mitigated by aircraft design and pilot training. As the key source of information for pilots, the human visual system has necessarily driven much of the evolution in cockpit technology. In contrast to the complicated, gauge-based systems of the past, the electronic flight displays of today's modern airliners are testament to advances in human factors engineering. The next step in flight instrumentation, although already used for some 50 years in the military, is just beginning to emerge in civil transport aircraft. Head-up displays (HUDs) allow pilots to see key flight instrumentation while viewing the outside world. The need to look down at the flight instruments is removed by the HUD, resulting in increased situational awareness and greater precision in aircraft control. While the head-up display is a welcome development, as with many instances of human-technology interface, the benefits provided by HUDs are not without potential drawbacks. This report is divided into two parts. Part A provides a thorough grounding in key areas of importance to the development and operation of HUD systems. Specifically, this includes an appraisal of the various facets of the human visual system, the history and development of conventional cockpit instrumentation, and a summary of the technical aspects and human factors considerations relating to HUD systems. This basis is built upon in Part B, which offers a more practical assessment of the HUD system used in a modern airliner, the Boeing 787. The report concludes with a number of recommendations that may be of use to an airline introducing HUD technology.

\section{Background information and literature review}

Vision in aviation: More than $90 \%$ of the information relevant to a pilot flying an aircraft is attained visually [1]. Development of the cockpit systems by which visual information is displayed to pilots has therefore relied on a sound understanding of the science of human vision. The following subsections outline the physiological makeup of the eye and the process by which visual information leads to perception of the environment.

Physiology of the human eye: From a functional perspective, the human eye acts much like a digital camera. At the front of the eye, the cornea and lens focus the image while the iris modulates the aperture (pupil) in response to the amount of available light and required focal length. The image is then detected by the retina and transmitted to the brain via the optic nerve. This subsection surveys the various anatomical aspects of the human eye that are of interest in an aviation context, particularly considering the human interface of a HUD system.

Focus and accommodation: In order for the eye to register a sharply focused image, certain structural alterations are required depending on the focal length or distance to the object of interest. The process of adapting focal length from a distant object to a near point is known as visual accommodation and involves three separate but coordinated functions - lens accommodation, pupil accommodation, and convergence [2]. The speed at which accommodation occurs varies between individuals and with age but it is generally a split-second affair [3]. The cornea and lens focus light to a point on the retina in a process called refraction (essentially the bending of light). While the bulk of the eye's refractive power comes from the cornea, its refractive index (level of refraction) is fixed. In order to vary the total level of refraction, the cilliary muscle inside the eye changes the shape of the lens to alter its refractive index [4]. Lens accommodation (near vision) involves contraction of the cilliary muscle around the lens which decreases its diameter resulting in a more spherical, highly refractive shape [2]. As people age, the lens becomes harder and therefore less able to be reshaped by the cilliary muscle, this causes presbyopia (long sightedness) which generally starts to affect people around age 40. In addition to changes in refractive index, the size of the pupil adjusts in response to both the focal distance and the amount of light. The adjustment of pupil size to cater for the level of ambient light is generally well understood; the pupil constricts in conditions of high illumination and dilates when ambient light is low. However, the pupil also has a part to play in visual accommodation. Utilizing the 'pin-

*Corresponding author: Ryan J Nichol, Massey University - College of Business, New Zealand, Tel: +6421970565; E-mail: ryan.nicholl@xtra.co.nz

Received March 09, 2015; Accepted April 27, 2015; Published May 03, 2015

Citation: Nichol RJ (2015) Airline Head-Up Display Systems: Human Factors Considerations. Int J Econ Manag Sci 4: 248. doi:10.4172/2162-6359.1000248

Copyright: ( 2015 Nichol RJ. This is an open-access article distributed under the terms of the Creative Commons Attribution License, which permits unrestricted use, distribution, and reproduction in any medium, provided the original author and source are credited. 
hole' effect, the pupil constricts during near accommodation to block light scattered by the periphery of the cornea and increase the depth of focus [2]. The functions discussed so far are sufficient for monocular accommodation; however varying the focal length of our binocular visual system requires one further aspect. As focus is shifted from distant vision to near accommodation, the eyes must angle inwards to track the point of focus. This movement, termed convergence, can be easily observed by having someone focus on an object such as a pen as it is moved towards the tip of their nose. Convergence occurs simultaneously with lens and pupil accommodation to complete the coordinated process [5]. It is important to note that the human visual system can only cater for one focal length at any point in time. Therefore, if focussing on a near object, the background will be out of focus and vice-versa. This has important implications for HUD systems which will be discussed in section four.

Photoreceptor cells and the retina: Photoreceptor cells located in the deepest layer of the retina at the back of the eye pick up light energy and convert this to an electromagnetic signal [6]. More specifically, when exposed to light, pigment molecules in the rod and cone receptor cells begin a series of reactions that result in the transmission of electrical signals through the optic nerve to the brain [7]. This process, known as photo transduction, involves a cascade of extremely rapid chemical transformations creating a signal that is sufficiently amplified for transmission to the brain [6]. An appreciation of the function and limitations of photoreceptor cells (rods and cones) is imperative when considering the role of vision in aviation. Although both are necessary for proper visual function, the roles and capabilities of each type of receptor are diverse. Table 1 outlines the differences between rods and cones (Table 1). In order to view an object (or specific part of an object) in normal light conditions, one would generally adopt an eye position such that the image is focussed in the centre of the retina. While this seems obvious, there is a physiological reason behind this tendency to look directly at an object of interest (Figure 1) rather than to one side. Responsible for the perception of detail, colour and motion, cone cells are found at much greater density in a small area of the retina known as the fovea, located in the centre, on the visual axis [8]. This central concentration of cones is the reason for the relatively narrow visual field they provide. The importance of cone cells for detail perception can easily be experienced by focusing on a point and surveying one's peripheral vision. Considering the sheer amount of information transiting the optic nerve to be processed by the brain, and the limitations of visual attention (discussed shortly), the restriction of high resolution imaging to the central visual field is intuitive .A simple analysis of the human visual system confirms our evolution as diurnal creatures. With cones shut down in very low light conditions the remaining vision (provided by rods) is colour less and of relatively low detail. Additionally, due to the lack of rods within the fovea, humans exhibit a night central blind-spot. In very low light conditions it is not possible to see a small, faint object (such as a star) by looking directly at it - instead, one must look to the side of the object causing the image to focus outside of the fovea, in a position of the retina containing rods [8].

\section{Visual perception}

Having discussed the processes by which visual information is acquired, it is appropriate to consider how it is then used by the brain to aid perception of the environment. Wickens and Flach [9] offer the following definition of perception as a component of information processing.

"perception involves the association of meaning to sensory stimulation. It is the process through which the marks on this page are associated with words and ideas. It is the process through which the geometry of the visual scene (e.g., apparent shape of the runway) is associated with states of the aircraft (e.g., above or below the correct glideslope)".

\section{Perception involves two main areas of interest [9]:}

- Signal detection - relates to the amount of energy or degree of change necessary for a stimulus or change to be detected. In terms of vision, this could involve the intensity and/or contrast of a source (energy), and the speed and/or extent of movement required for the detection of motion (change).

- Selection - describes the way humans attend to and extract relevant information from the enormous amount of stimulus detected. This area is of particular interest when considering HUD systems, which present information in a way that it is highly salient, and likely to be selected above other stimuli (discussed further in Section 4). Perception may consist of components that are derived from the external environment only (ecological perception), or more often in aviation, components that are derived from the environment and augmented by individual experience through comparison with memory and expectations [10]. Learning to flying and gaining experience is in part an exercise in developing a memory bank of prior experiences and training from which pilots will compare sensed information, resulting in environmental perception. An example of this process is learning the various facets of runway perspective on approach for judging glideslope - readers will recognise this knowledge as something that is substantially gained from experience and training.

Recognition and orientation: Leibowitz [8] discusses two modes of visual processing, object recognition and visual guidance, and explains that each utilises completely different parts of the brain. Leibowitz [8] points out how this dual functionality is evident in the process of reading while walking - humans are able to simultaneously exercise the functions of pattern recognition (recognising words in a book, or on a cell phone) and spatial guidance (walking), even though attention is almost completely dominated by the former. If one considers human evolution, it makes sense that guidance during self-locomotion (moving around by walking or running) is largely automatic. This frees up our attention for primitive tasks involving recognition, such as hunting or fighting. Importantly, the tendency for humans to rely on automatic visual guidance transfers to the operation of modern transportation [8]. Considering the example of driving a car, it is generally well understood that although intuitive, our ability to move at high speed without direct attention while operating a vehicle is detrimental to safety, hence modern legislation against the use of cell phones while driving. The fact that hands-free phones are almost as dangerous while driving as hand-held types further emphasizes the role of attention, rather than mere physical distraction [11].

\begin{tabular}{|l|l|l|}
\hline & Rods & Cones \\
\hline Level of illumination required & Low & $\begin{array}{l}\text { High (deactivated in } \\
\text { low light) }\end{array}$ \\
\hline Detail perception and quality of vision & Inferior & Superior \\
\hline Colour perception & No & Yes \\
\hline Motion detection & Superior & Inferior \\
\hline Visual field & $\begin{array}{l}\text { Wide } \\
\text { (peripheralvision) }\end{array}$ & $\begin{array}{l}\text { Narrow (small amount } \\
\text { in periphery) }\end{array}$ \\
\hline
\end{tabular}

Table 1: Differentiation of photoreceptors. 


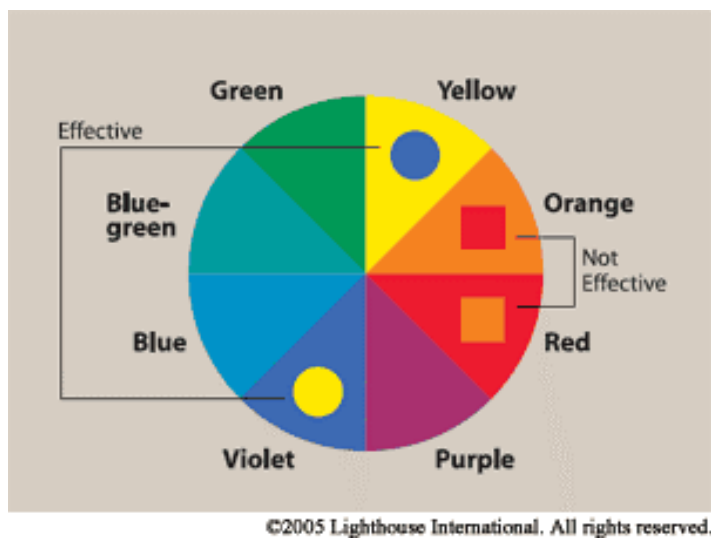

Figure 1: Hue contrast (Source: Lighthouse International).

The dangers of inattention and overconfidence in visual capabilities are compounded at night due to a reduction in relevant stimuli. A wellknown phenomenon in aviation, the black-hole illusion is a direct result of humans relying on automatic response to certain visual cues that are misleading due to the artificial environment (high speed, elevation above the ground). The tendency of pilots to believe they are higher than they actually are on approach under conditions of sparse ground illumination highlights the problem that can occur in relying on a perception system that was not designed to encounter such situations. The black-hole illusion also acts as an example of how such pitfalls may be mitigated. Gibb et al. [10] explain that while knowledge of the black-hole effect in itself is not sufficient, simulator training designed to reduce pilot confidence in their visual capabilities is of benefit. The black-hole illusion is now an area of specific training for pilots, focusing on the need to supplement visual runway perspective with glidepath guidance and altitude-distance crosschecking. This seems to have reduced the incidence of related occurrences. There are many other examples of perceptual illusion in the aviation environment. For the purpose of this report, the focus is not so much the specific types of illusion, but the understanding that the human visual system has limitations when operating in the aviation environment that must be understood and mitigated by human factors engineers and operational training programs.

Visual attention: Visual attention is the process of selecting a location (or locations) in the visual scene that are of relevance to the task at hand. Attentional control can be based on either stimulus considerations, such as the salience of an object or region, or goal relevance, such as how closely an object matches the target being searched for [12]. The visual working memory (VWM) supports several visual representations for a brief period, and modulates competition for attention between objects in the visual field [13]. It has been widely accepted that prioritization of information in the VMW requires sustained visual attention [14-16]. In contrast to the extant literature, research by [13]. Suggests that information can be prioritized for retention in the VWM even without sustained visual attention, although the authors note their results do not preclude prioritization by visual attention. Of relevance to HUD systems, Hollingworth and Maxcey-Richard also found that "prioritization remained robust when spatially overlapping memory items precluded selection by means of attending to a particular location", which suggests that colocated information such as HUD symbology and the background can be selectively attended to. Conversely, Ververs and Wickens [17] note that "the colocation of attributes in perceptual space which supports a divided attention task may also make it difficult to focus attention on one particular item and filter the presence of the others". These ideas will be built-on with evidence suggesting attention capture by HUD systems in Section 4.

The ability to attend to a limited amount of information at one time has significant implications for aviation. Piloting an aircraft presents a situation where numerous objects compete for attention. As discussed above, the visual working memory acts to meter out attention to competing stimuli. As part of this process, focusing on specific information causes the inhibition of responses to other stimuli, potentially resulting in in attentional blindness [10]. This ability to focus on certain aspects of a scene while blocking out others is essential to human operation in complex environments but also potentially dangerous when flying an aircraft. In order to efficiently attend to various information sources, and appropriately balance their time between focused and divided attention, pilots are taught the process of 'scanning', or attending briefly to each information source sequentially in a systematic fashion [18]. The HUD system reduces the need to scan between the outside view and cockpit instruments, perhaps bolstering the case for attention capture, i.e. the reduced ability to prioritize attention between competing stimuli in the VWM. It is clear that much is still to be discovered about the nuances of visual attention, and this must be borne in mind when considering the use of head-up displays.

Colour and contrast: Contrast affects the signal detection component of perception. Simultaneous contrast is the comparative colour and luminance/brightness of adjacent objects in the same field of view, such as the words on this page against the background (or indeed, HUD symbology against the outside view). The human visual system is more sensitive to contrast than absolute luminance, allowing for similar perception of objects over a wide range of lighting conditions [19]. In practical terms, it is easily understood that the high contrast arrangement of black text on a white background (or viceversa) is more easily read than if text and background were two similar shades of grey. In a dynamic visual situation, successive contrast refers to the effect on the perception of an object by the previous viewing of another object of different colour/intensity [20]. An example of successive contrast would be transferring visual attention between a bright cockpit display and a dull outside scene on a rainy day, resulting in the reduced salience of subtle visual cues in the outside world. The addition of colour to perception of the visual environment adds another dimension. As previously discussed, cone cells in the retina are responsible for detecting colour. In fact, there are three different types of cone (red, green and blue), each responsible for the detection of a different wavelength. While the initial determination of colour is physiological, interesting work by Williams [21] has shifted understanding of colour perception to more of a cognitive function rather than being purely photoreceptor-based. Williams found that although there is large variability in the number and density of each type of cone cell between individuals, perception of colour in terms of matching a perceived colour to a specific wavelength of light, is surprisingly uniform and can actually be recalibrated in either direction over time. An example of training or recalibrating colour perception occurs when a donning a pair of sunglasses with coloured lenses - the initial result is a conscious skew in one's perception of environmental colours, but this dissipates with time as the brain recalibrates and the filtering effect of the lenses may even be forgotten.

Although slightly more complex than for greyscale (black and white), the contrast between different colours is still relatively intuitive [22]. Figure 1 illustrates how opposite colours such as violet and yellow 
provide for higher contrast than similar hues such as red and orange. The depth of shade or saturation is also important, lighter shades contrast more strongly with darker shades as shown in Figure 2 [23]. The use of colour is important in aircraft displays due to the representative nature of different hues, much like the role of symbols in supplying information concisely and efficiently. For example, artificial horizons (and their derivatives) normally represent the sky with blue colouring and the ground with a brown colour. Various navigation symbology is also colour coded to aid pilot's situational awareness, and caution and warning indications relating to system operation are represented by familiar yellow/orange and red respectively. Conversely, green is generally understood to denote safety, or the normal operation of a system. Considering its culturally-assigned meaning, it is important to realize that the green colour of a HUD display is likely to make the recognition of system caution or warning information less immediate. The use of bold and flashing symbology goes some way to mitigating this shortcoming.

\section{Conventional Flight Instrumentation}

Supplying pilots with adequate, meaningful and ergonomicallydisplayed information about the status and operation of their aircraft is critical in mitigating human perceptual limitations. From the humble beginnings of the Wright Flyer, where instrumentation consisted of a stop watch, tachometer and anemometer, the last century has seen tremendous developments in the area of flight instrumentation. This section provides a brief overview of historical developments and broad appraisal of the modern head-down primary flight display.

\section{History of Flight Instrumentation}

As technology, human factors knowledge, and indeed the aeroplane itself have all evolved, so too has instrumentation in the cockpit. Focusing specifically on civil transport aircraft, Jukes [23] classifies this development into three eras, summarized in the following subsections.

The mechanical era: The Wright Brothers understood the need to visually represent important information. An early example still used today by many glider pilots, is the placement of a piece of cloth or string in the airflow to indicate slip/skid during turns. An evolution of this, the 'balance ball' is a purely mechanical instrument still used in many modern aircraft. The mechanical era was defined by the direct use or development of existing scientific instruments, such as the wind anemometer for airspeed, weather vane for angle of attack, and barometer for altitude. The first paper specifically addressing aircraft instrumentation was written in 1912, marking the beginning of work in this constantly evolving field.

The electromechanical era: While the purely mechanical design of pressure-based instruments such as the altimeter was still adequate for slow, low altitude aircraft, the push towards 'flying blind' by USAF required development of electrically powered navigation instruments. The crude radio-guidance system used by Doolittle marks a key point in the evolution of flight instrumentation towards the ground based navigation systems of today, themselves being progressively phased out in favour of satellite and aircraft-based systems. The instrument flying concept also necessitated development in the area of gyroscopic instrumentation, specifically the artificial horizon and directional gyro which were pneumatically driven. Eventually, pressure-based speed and altitude instrumentation on fast, high-speed civil transports also joined the list of electromechanical instruments, and pneumatic gyro instruments developed into electrically powered inertial reference systems. With this rapid expansion in the amount of displayed information, thought was needed into the placement of each instrument in order to maximise utility to the pilot. From the early 1930's, aircraft designers began to use various iterations of the 'basic six' arrangement of key flight instruments (Figure 3) and many light aircraft still use this setup in a slightly modernised format sometimes termed the 'six pack' (Figure 4). In an environment of faster, more mano euvrable aircraft, and with the benefit of experience and advances in human factors knowledge, the US Air Force formed a team of engineers, pilots and human factors experts in the late 1950's with the goal of redesigning the instrument panel. The advances made by the military were quickly appraised by civil aircraft designers, leading to universal adoption of the 'basic T' layout on electromechanically instrumented civil transport aircraft (Figure 5).

The electro-optical era: Developments in computer processing and cathode ray tube (CRT) display technology were first applied to civil aircraft in the mid 1970's as part of the supersonic transport program and later the NASA terminal configured vehicle program using a Boeing 737. In parallel to the US research, British Aerospace Corporation undertook work leading to the first flight of an 'advanced civil flight deck' equipped BAC 1-11 demonstrator in 1981. The BAe flight deck boasted an electronic flight instrumentation system (EFIS) consisting of two colour CRT displays configured side-by-side in the form of the now familiar primary flight display (PFD) and navigation display (ND). The PFD incorporates an artificial horizon and flight director with an airspeed indicator and altimeter (Figure 7). The first production civil transports to use a 'glass' flight deck were the Boeing 767 and 757 which entered service in September 1982. The 757/767 retained the ' $T$ ' flight instrument layout (Figure 6), comprising an electronic attitude director indicator (EADI) (flanked by electromagnetic airspeed indicator and altimeter) and an electronic horizontal situation indicator (EHSI) [24]. The fully-integrated side-by-side PFD/ND setup was not seen in service until the launch of the Airbus A320 in 1988 (Figure 7) but quickly

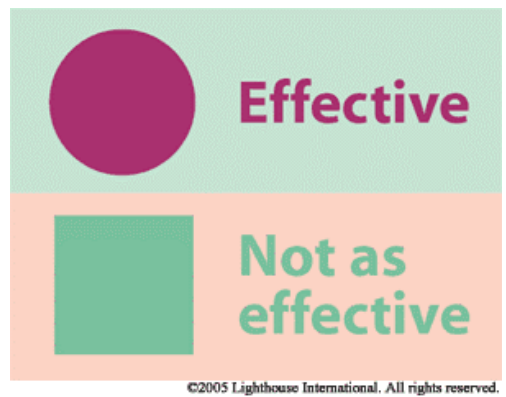

Figure 2: Saturation contrast (Source: Lighthouse International).

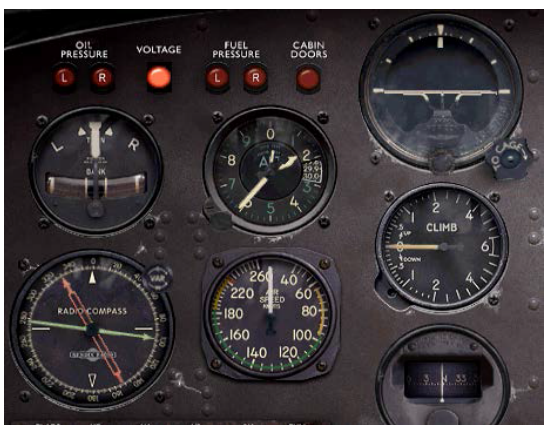

Figure 3: Early 'basic six' arrangement - Douglas DC-3 (Source: MS Flight Simulator $\mathrm{X}$ ). 
Citation: Nichol RJ (2015) Airline Head-Up Display Systems: Human Factors Considerations. Int J Econ Manag Sci 4: 248. doi:10.4172/21626359.1000248

Page 5 of 12

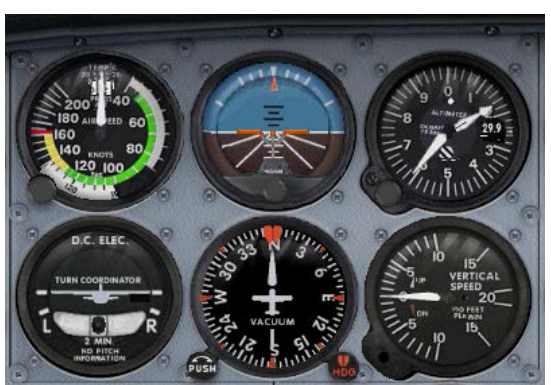

Figure 4: Modern 'six pack' of basic flight instruments - Cessna 172 (Source: MS Flight Simulator X).

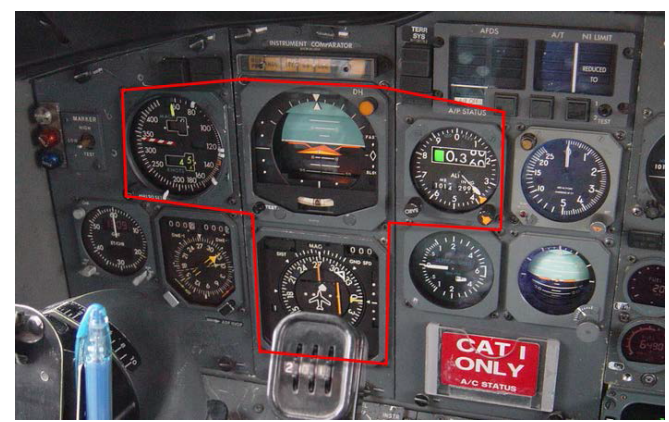

Figure 5: Boeing 737 panel showing 'basic T' configuration (Source: AviaFilms.com).

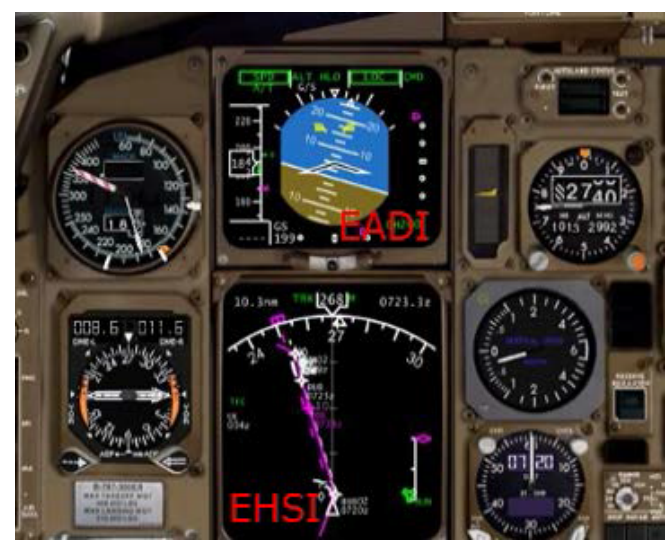

Figure 6: 757/767 panel (Source: barnestormers.com).

became (and continues to be) the standard layout for new designs. Reference information for modern EFIS displays generally comes from a solid state (rather than gyroscopic) inertial reference system, giving increased accuracy and reliability. CRTs have been superseded by flat panel active-matrix liquid crystal displays (AMLCD), first used in the Boeing 777 as part of its integrated avionics architecture.

The primary flight display: This subsection will briefly survey the current state of the art with respect to primary flight instrumentation. A Boeing 787 PFD is used as an example, however a simple comparison shows that the information and layout is similar to even the early Airbus PFD in Figure 7. The only real difference between the 787 PFD and earlier versions is its full-span artificial horizon (which extends behind the speed and altitude tapes), otherwise it is substantially similar to those used in the 777 and 737NG (600-900 series). A further discussion of PFD components including colour and symbiology is given in Part $\mathrm{B}$ of this report.

\section{Description of Displayed Information [25]:}

1. Flight mode annunciations: confirms the current auto flight speed, lateral navigation and vertical navigation modes.

2. Speed/mach indications: the speed tape moves vertically behind the fixed digital readout which displays current indicated airspeed from the air data reference system (ADRS). The magenta bug shows the commanded speed, which is also represented digitally at the top of the tape.

3. Artificial horizon and flight director: displays attitude information derived from the inertial reference system (IRS) and flight director commands (magenta bars).

4. Autopilot/flight director system status.

5. Altitude indications: works in the same way as the speed tape, with ADRS altitude information displayed. Selected QNH is displayed in green below the tape.

6. Vertical speed indication: derived from the ADRS, the placement allows visual interaction with the altitude tape bug, i.e. during level off.

7. Miniature map: displays selected IRS navigation information such as heading and track in a condensed format (full information is given on the adjacent navigation display panel).

The information displayed on a PFD was previously spread among several different instruments. Without going (Figure 8) into great detail, it is not difficult to appreciate the benefits gained by integrating the various associated information into one display. Development of the PFD provides a highly visible example of the extent to which ergonomics and other human factors have played a part in the evolution of aircraft design.

\section{Head-up Display Systems}

The primary flight displays of modern transport aircraft do an excellent job of presenting information to pilots in a way that promotes efficiency and good situational awareness. However, the need to transition from the use of head-down displays to outside visual reference at certain points in the flight continues to create an attentional division, often during critical management periods. The use of head-up displays (HUD) brings primary flight management information and outside visual reference into the same visual scene, increasing the usefulness and relevance of displayed symbology. This section surveys the

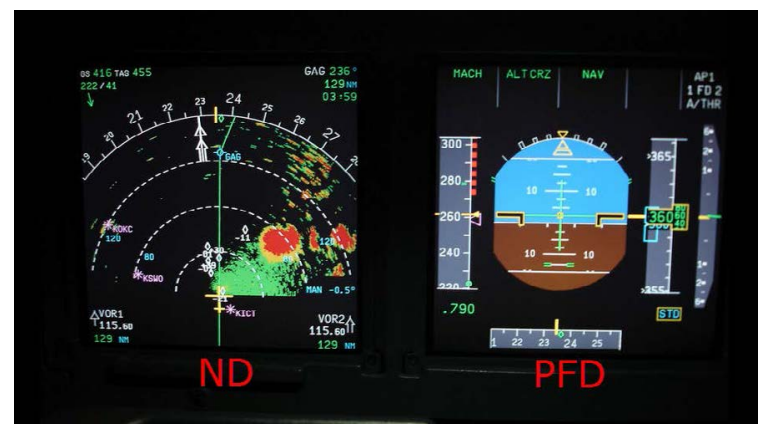

Figure 7: Airbus A320 co-pilot side integrated flight instrument system (Source: Knut Hansen). 


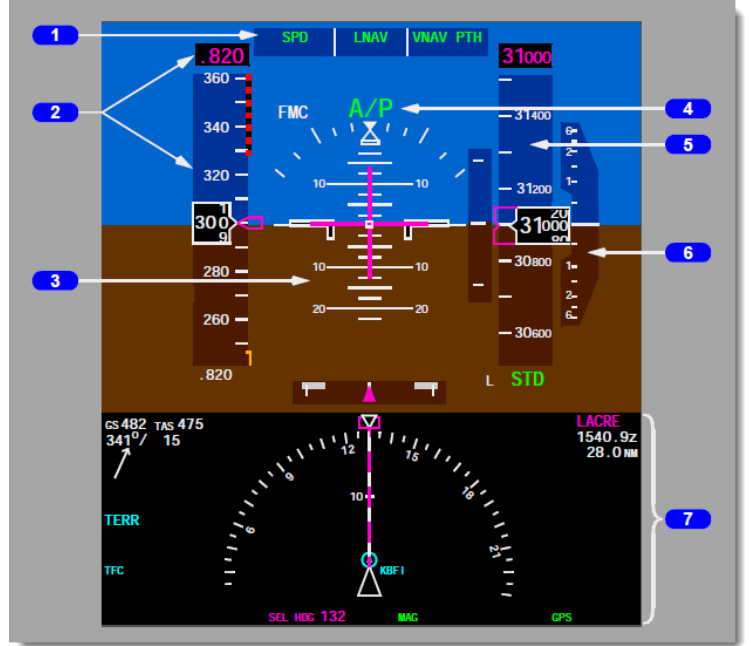

Figure 8: Boeing 787 PFD (Source: Boeing)

historical development of the HUD, the components and technology that comprise a HUD system, and the benefits and limitations of HUD technology.

\section{History of the HUD}

Like much aviation technology, the head-up display has military foundations. The initial concept was first seen in the reflector gunsight, developed by Sir Howard Grubb and used in fighter aircraft of the 1920 's. The mirror in this simple display was later augmented by gyroscopic input to project a gunsight with a lead angle adjusted for speed, altitude, attitude and turn-rate [25]. The gyro gunsight entered service aboard RAF Spitfires and Hurricanes in 1943 (Figure 9). The idea of supplementing gyroscopic sights with other conformal (aligned with the outside view) and trajectory related symbology, particularly the flight path vector, came about in the 1950s and in 1962 the first HUD-equipped Royal Navy Buccaneer went into service [26]. HUDs became commonplace in military strike aircraft of the 1970s, and more recent fighter jets make use of helmet mounted displays (HMD) offering greater display flexibility in their high visibility cockpits [27]. Although accurate weapons aiming drove HUD development in the military, the ability to improve situational awareness by providing pilots with all kinds of information while keeping their eyes outside meant it wasn't long before civilian applications began to emerge. Single (captain side) HUD systems have been optional for a number of years on commercial transport aircraft such as the Embraer 190, Boeing 737 and more recently, certain Airbus aircraft. The Boeing 787 is the first large commercial aircraft to offer a HUD as standard equipment, utilising a dual Rockwell Collins head up guidance system. The next generation of HUD technology adds synthetic terrain or infrared video information to further enhance the display (Figure 10).

\section{Anatomy of a Civil HUD System}

A head-up display system works by projecting symbology onto a transparent, semi-reflective screen (called a combiner) in front of the pilot, allowing them to monitor primary flight information while viewing the outside world [28]. This subsection will provide a thorough description of the major design aspects of a modern civil transport HUD system. Head-up displays consist of two main components, the projector and the combiner (Figure 11), discussed here in turn. The actual image displayed will be described and compared with the PFD in Part B of this report. Generation of the HUD image is essentially the same as (although redundant from) head-down displays, so will not be specifically discussed here.

Collimated image: As discussed in Section 2, the human eye is able achieve only one focal length at a time. One of the key principles enabling head-up displays is the creation of an image at optical infinity, meaning the rays of light are parallel (collimated), rather than divergent as for a close-up image. This principle is also used in modern flight simulators to give the perception of depth to the simulated outside view. Any visual scene formed at a focal length of six meters or greater is said to approximate optical infinity [29] meaning the collimated image provided by a HUD allows the pilot to view the outside world and HUD image in focus concurrently.

There are two means of generating the collimated HUD image [23]

- Collimating HUD (refractive) - the image is fully collimated by refraction in the projector lens system and reflected by a flat combiner. This results in a limited instantaneous field of view, meaning the pilot must move their head around to see the total image (Figure 12).

- Pupil-forming HUD (reflective) - the projector lens only partially collimates the image. The remaining angular change is induced by a curved (convex) combiner (Figure 13), resulting in a field of view that is limited only by the size of the combiner. Current civil transport HUDs all utilise reflective systems

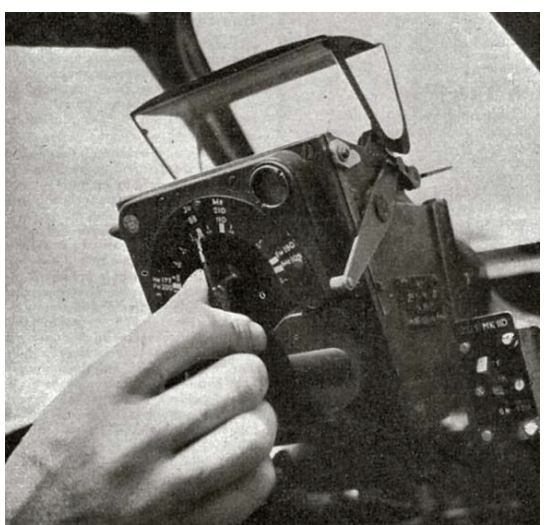

Figure 9: Mark II Gyro Sight aboard Spitfire (Source: Wings magazine).

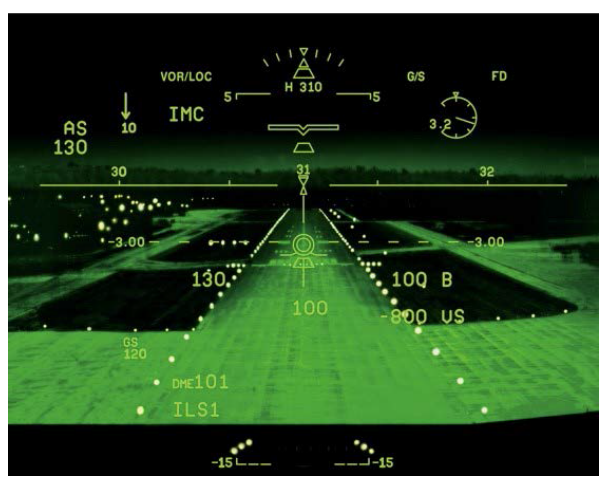

Figure 10: Rockwell Collins HGS with Enhanced Visual System (Source: Boeing). 


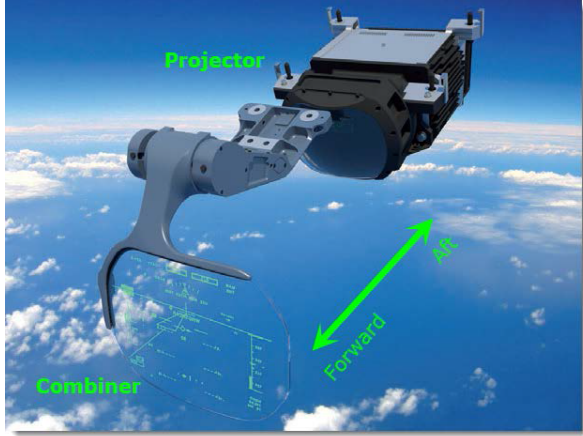

Figure 11: Modern Airbus HUD system (Source: Thales Avionics).

which are more useful as a primary flight reference due to their larger instantaneous fields of view [30].

While it is true that HUDs physically allow the two different scenes to be viewed, there is debate about the level to which pilots can actually look at or attend to both images at the same time, this point will be elaborated on shortly.

The projector: Initial HUD projectors used a CRT source, generally containing P-53 phosphor which generates a narrow spectral emission peak around $545 \mathrm{~nm}$ (green). The narrow wavelength band is imperative to the operation of the HUD (discussed next), and the green colour around $550 \mathrm{~nm}$ falls at the peak of the eye's photopic response (the green curve in Figure 14), meaning it is generally the most effective colour for detection [23]. Modern systems use an LED light source modulated by an LCD screen which results in even greater wavelength specificity, as well as increases in operational efficiency due to lower power use and reduced maintenance. The intensity of the display can be adjusted in response to background lighting and contrast effects either manually, or in modern units, using automatic contrast sensing.

The combiner: As its name suggests, the role of the combiner is to display the reflected HUD image while allowing a degree of transparency to the outside world. The combiner in a pupil-forming system is also responsible for altering the angle of the reflected image, without creating any noticeable refraction of the outside view. In order to differentiate these opposing tasks, the combiner must be reflective to the monochromatic HUD image, but largely transparent to everything else - this can be achieved by a number of different fabrication processes. Because the combiner is designed to reflect only a very specific band of light, the narrow wavelength peak provided by the HUD projector is important. Modern systems boast reflection of nearly $80 \%$ of the projected image, with only $20 \%$ attenuation of the outside view [23]. Although the technology exists to create the HUD image directly in the combiner using optical waveguide technology [31], this type of system is yet to be used in commercial aircraft.

Head motion box and field of view: HUD systems require pilots to adopt an accurate and consistent eye position in order to view the display fully. The head motion box, or 'eyebox' is centred around the ergonomically positioned design eye point (DEP), which gives the best trade-off between HUD and other cockpit visual tasks. The eyebox dimensions in modern pupil forming systems are approximately 130 $\mathrm{mm}$ laterally, $75 \mathrm{~mm}$ vertically and $150 \mathrm{~mm}$ longitudinally [30]. An added benefit of HUD use is that pilots are forced to adopt the eye position intended by aircraft designers, often not the case when left to their own devices. The concept of field of view (FOV) was introduced previously, but warrants further discussion. One of the key aspects of
HUDs is that they display information over a wide angle compared to head-down instruments, this may be compared to viewing a movie screen versus a television. Although the actual HUD combiner is similar in size to a PFD screen, proximity to the pilot's face creates a relatively wide angle display. Pupil-forming HUDs boast a total FOV in the order of 22-28 degrees vertically and 28-34 degrees horizontally [30], compared to the roughly 12 degree FOV displayed on a PFD. The implications for flying accuracy may be apparent at this point, but will be elaborated on in Section 5. It should also be noted that the wide FOV of a HUD necessitates a larger scan pattern (greater eye movement), which some pilots may find tiring at first.

\section{Benefits of HUD technology}

Head-up displays are credited with various benefits, including

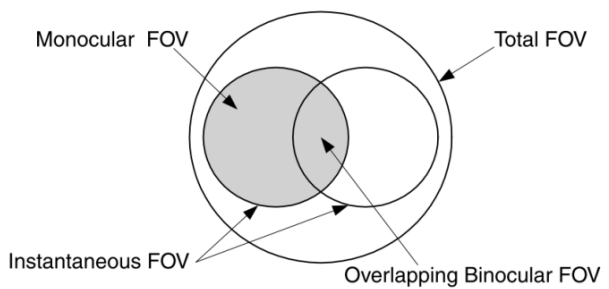

Figure 12: HUD fields of view (Source: Woods \& Howells).

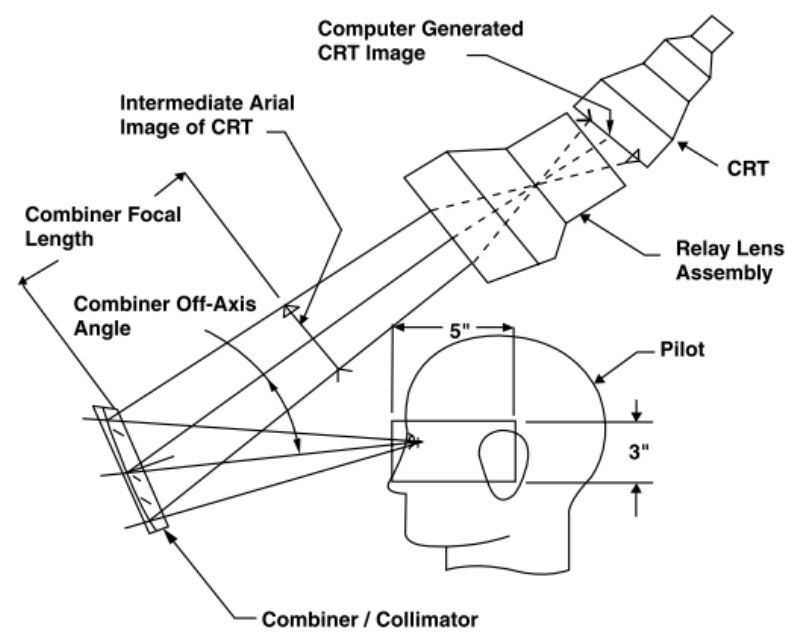

Figure 13: Pupil-forming HUD (Source: Woods \& Howells).

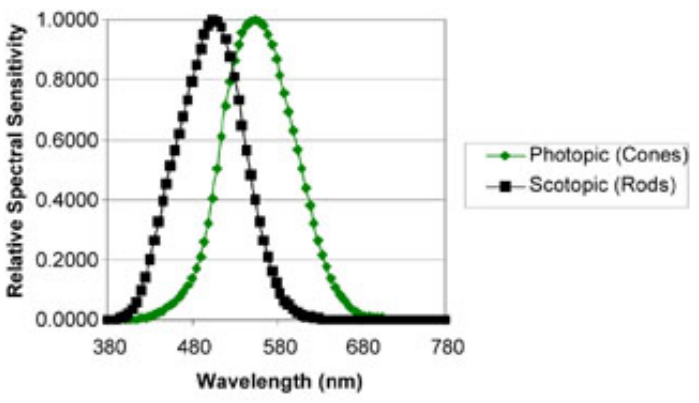

Figure 14: Photopic response (Source: SPIE). 
improving track and glide path maintenance on approach [28], improving landing precision, reducing the required visibility for takeoff and landing, increasing situational awareness [32], and even assisting flight crews suffering smoke in the cockpit [33]. The HUD is generally regarded as a positive contributor to flight management and it has been proven empirically that HUDs improve detection of expected events (both on the display and beyond) and routine flight control making them clearly superior to their head-down counterparts [34]. The benefits of HUD use are reasonably intuitive, so warrant limited discussion here. A greater focus will be placed on potential shortcomings in the next subsection.

\section{Limitations of HUD technology}

Although the net benefit of HUD use is overwhelmingly positive, like many aspects of aircraft technology there are certain potential drawbacks relating to the interface between human and machine. A survey of the literature points to attention capture as the main issue of importance to operators of HUD-equipped aircraft. It is not the goal of this report to debunk the usefulness of HUD, rather to emphasise areas requiring greater training focus in order to ensure crews realise the full benefits of the technology.

Attention capture/cognitive tunnelling: Attention capture or cognitive tunnelling describes the potential for highly salient information such as head-up display symbology to monopolise attention at the expense of the less invasive background scene. Although HUDs do a good job of presenting information in a way that can be viewed simultaneously with the background, the attentional limitations discussed in Section 2 affect the way pilots process co-located HUD and background information in various different environments and under varying degrees of workload. Brief mention is made of the possibility for HUD attention capture in the following excerpt from the Boeing 787 FCTM [1] New HUD users may notice a tendency to focus attention on one layer of information (e.g., the HUD symbology) at the expense of the ther (e.g., the outside environment). The following techniques will help crews to gain the best use from the HUD:

- adjust the brightness so the pilot can see the symbology on the HUD and can see through it

- the PF looks through the HUD symbology to use normal outside cues

- the PM uses a continual scan technique

- pilots will be less susceptible the more they use the HUD and practice the attention shifting techniques.

In addition to the above techniques, modern HUDs also offer a 'de-clutter' (reduced display) feature, designed to minimise the chance of distraction and attention capture during critical phases such as takeoff and landing. The pilot may choose between de-clutter and standard mode at any stage, generally using a button on the control column. Boeing's assertion that the attention capture (also known as cognitive tunnelling) problem is limited to inexperienced HUD users is not necessarily backed by evidence, although [35] do suggest an experiential component, and offer that appropriate training combined with experience may mitigate the risk. As with the black-hole illusion example in Section 2, appropriate training is clearly an important component in the minimisation of HUD attention capture and related risks. A potential training option suggested by [36] aims to increase scanning effectiveness by teaching pilots to take their attention away from the HUD and focus on the far domain, although [37] highlight the need for further development in the area of HUD attention training. Cognitive tunnelling caused by head-up displays has been a concern since the effect was first discovered by [38] who found that pilots using head-up displays were less likely to detect an unexpected runway incursion than those flying with conventional instruments. The authors noted that HUDs generally preserve the runway view within foveal vision, leading to an attention-based explanation for this deficiency [39] explain that "the HUD can act as an attentional 'trap' that draws information processing resources to the HUD and slows/degrades processing of external events" (p. 1). It is suggested that division of attention between two overlapping sources is difficult and unnatural [40]. Further, the use of dual frames of reference makes accurate and complete information gathering problematic [37]. The effect of cognitive tunnelling on event detection is moderated by the expectancy and saliency of the event [35] find that cognitive tunnelling only affects detection when an event is not very salient and truly surprising - for example a small vehicle such as a baggage cart blocking the runway. On a related tack, [39] consider the effect of contrast ratio on the detection of events both on the HUD symbology and in the outside scene. The importance of contrast over absolute luminance was discussed in Section 2. Figure 15 clearly shows how a contrast ratio of three or four gives the best trade-off, with higher ratios (brighter HUD) leading to more outside events being missed and vice-versa. One of the common components of nearly all published HUD research is the use of flight simulators rather than actual aircraft. While flight simulators are adequate in many respects, it must be noted that this artificial environment has the potential to affect results. It would be useful for airlines to develop high fidelity reporting systems to allow for the capture of real-life data relating to airline HUD use. In any case, it is clear that room exists for further research into the use of head-up displays in an airline environment [41].

Diverse background contrast: The previous subsection touched on the narrow range of optimum contrast ratios between HUD and background. Although HUD brightness can be adjusted (manually or automatically) for changes in ambient lighting and background contrast ratio, current systems only allow for one brightness setting over the entire image, moderated at the projector unit. Figure 16 provides a clear example of how diverse background colour and lighting affect contrast (note the difference in contrast and therefore readability between sky and ground backgrounds). Another example of this situation would be a night approach to a runway with bright city lights on one side, and a body of water on the other. while there doesn't seem to be a solution to this problem at present (or even a great degree of acknowledgement that it is in fact a problem), one possibility that comes to mind is the development of a combiner with variable reflectivity based on background contrast, or the division of the projected image into a number of sectors capable of different luminance levels (based on automatic contrast sensing). Alternatively, a move to optical waveguide-based systems would offer a simpler solution contained within the combiner - perhaps an area for further study.

Turbulence: The fact that most HUD research has been conducted in simulators has perhaps led to the effect of turbulence being overlooked. Although completely anecdotal, discussions with pilots using HUD in Boeing 737-800 aircraft on approach to Wellington in turbulent conditions suggests that many find the use of HUD difficult, sometimes leading them to stow the combiner and revert to traditional head-down displays. The Boeing 787 FCTM does note the potential for turbulence and the resulting large control inputs to magnify movement 


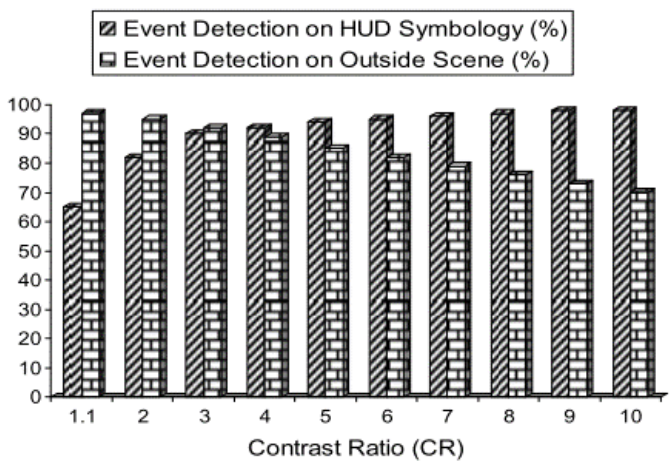

Figure 15: Effect of contrast on event detection (Source: Karar et al.)

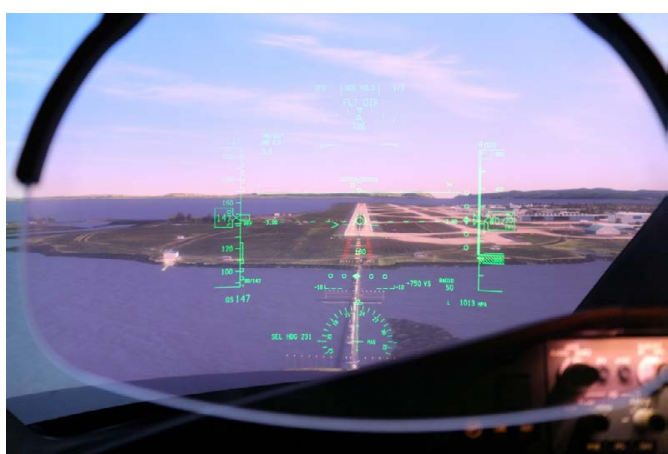

Figure 16: Boeing 787 (simulator) HUD showing background contrast difference (sky and ground).

of the flight path vector. It is possible that this is mainly an issue for newer HUD users who may revert to more familiar systems in difficult conditions. Further study is required in this area, adding weight to the need for an appropriate QA reporting system.

\section{Practical analysis - Boeing 787 HUD}

\section{Boeing 787 simulator - practical HUD analysis}

This section will build on the knowledge from Part A with a practical analysis of the Boeing 787 dual head-up display system, which is standard on all 787 aircraft. The Air New Zealand 787-8 simulator is used to gain a broad appreciation of HUD use. While limited inference can be drawn from this short exercise, it does nevertheless provide several points for discussion.

\section{Comparing the HUD with the PFD}

The following information should be read in conjunction with the Boeing 787 FCOM.

General comparison: Figure 17 shows the HUD and PFD during a 30 degree turn to the left, level at 4000 feet and three knots below the commanded speed of 280 knots. Perhaps the most obvious point to note is the similarity of both displays. Both contain almost identical speed and altitude tapes (although vertical speed is only given numerically on the HUD). The flight mode annunciators (FMA), autoflight annunciator and bank angle scale are also substantially similar (it appears the autopilot has been engaged while the simulator was paused before the PFD shot). The concept of an expanded field of view was discussed in Section 4. One of the limitations of artificial horizon-type instruments is the fact that the angular scale is compressed. It can be seen that the $+/-10$ degree pitch reference lines are only just visible at the top and bottom of the HUD, compare this to the PFD which shows +/- 20 degrees, both show the current pitch of the aircraft at two and a half degrees nose up. It should be noted that the horizon line displayed on the HUD appears increasingly above the visual horizon as altitude is increased, due to the curvature of the Earth. The heading scale on the HUD horizon is also expanded compared to that of the PFD/ND. Figure 17 shows a current heading of 330 degrees, with 320 and 340 at either end of the horizon - compare this to a 20 degree span on the traditional compass rose at the bottom of the HUD and PFD (Figure 18). It is the combination of expanded scale and flight path vector (discussed shortly) that make for far greater manual flying accuracy with the HUD.

Monochrome display: One of the major developments in flight instrumentation over the years has been the use of colour to denote various different types of information. For example, magenta coloured symbols on the PFD are instantly recognised by pilots as commanded or 'target' parameters selected via the mode control panel and/or flight management system - this includes commanded speed and altitude (both the bug symbols at the centre of the speed and altitude tapes, and the digital displays at the top). Additionally, Figure 18 shows how red and yellow are used to denote information of immediate importance to safety - examples include the red 'bricks' on the speed tape indicating minimum and maximum speeds for the current configuration, and the yellow symbols on the altitude tape indicating a 'below minimum altitude' condition. As discussed in Section 4, HUDs necessarily use a single-colour (green) display. This results in the removal of a layer of information normally provided by colour coding. While the use of identical symbology and similar layout mitigates this somewhat, lack of colour is nevertheless something that pilots will take some time to adjust to. The fact that HUD use has been shown to improve situational awareness and flying accuracy indicates that the lack of colour is more than outweighed by positive factors.

The flight path vector: Figure 19 shows the following symbology

1. Flight director guidance cue

2. Flight path vector (FPV)

3. Horizon line heading scale

The FPV differs from the fixed 'airplane symbol' (pictured above the guidance cue) in that its position changes relative to the projected path of the aircraft as derived from the inertial reference system. In basic terms, the vertical position of the FPV represents the climb/ decent angle with reference to the pitch scale, e.g. if the centre of the FPV is on the horizon line as in Figure 17, the aircraft is flying level (Figure 19 shows an approximate one degree climb angle). The horizontal position denotes drift, in still air and balanced flight, the FPV will be positioned in line with the centre of the airplane symbol (at a 90 degree angle to the horizon). Any drift will result in the FPV being offset from the centre of the airplane symbol and the projected track of the aircraft can be devised by comparison to the heading scale on the horizon line. Figure 19 shows the aircraft heading 047 degrees (pointer beneath airplane symbol) and tracking about one degree right of that (FPV). Consideration of a visual approach provides an intuitive and practical appraisal of how the FPV is used operationally. Approach to land requires stable velocity in both vertical and horizontal planes. Once lined up with the runway and on glidepath, the pilot simply points the FPV at the touchdown zone. In order to adjust track or glidepath, the FPV is moved in the desired direction until the 


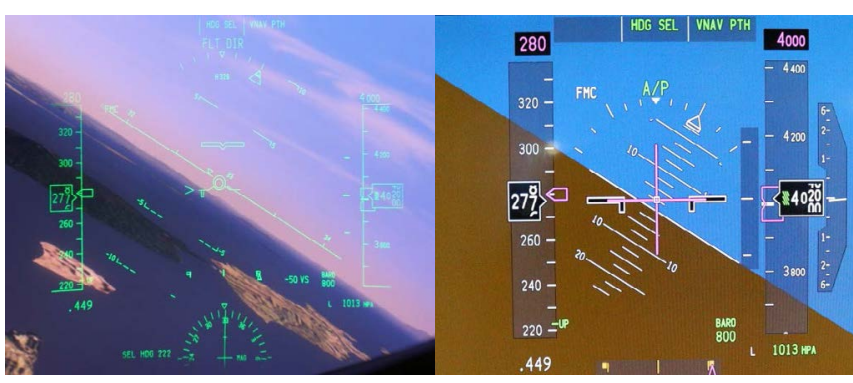

Figure 17: Boeing 787 HUD and PFD during a level turn.

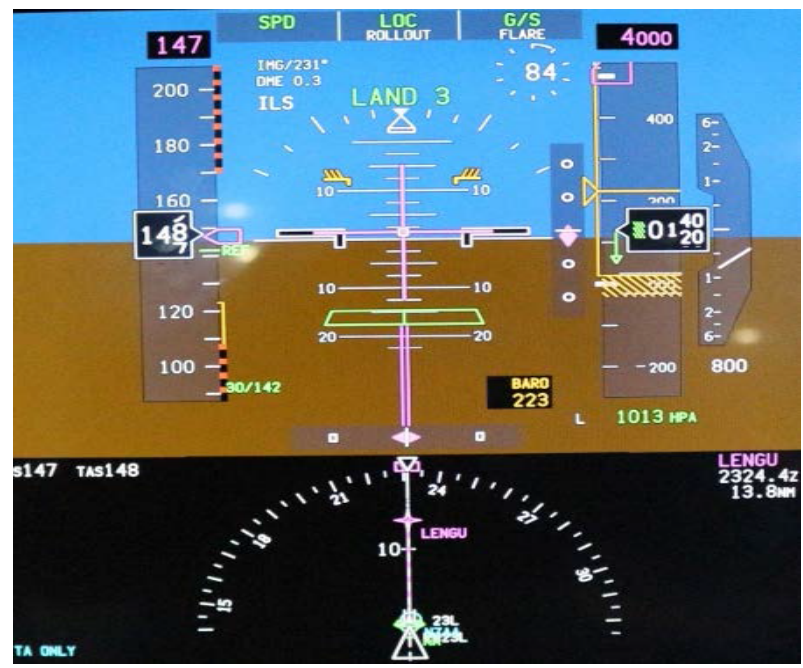

Figure 18: PFD colour-coding.

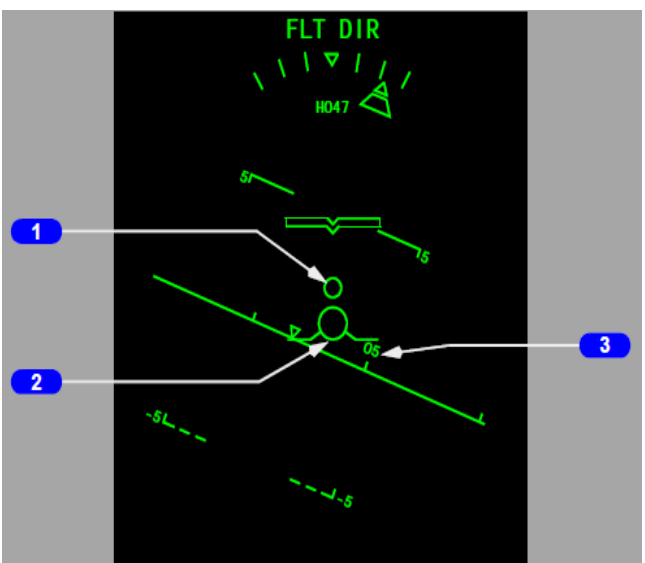

Figure 19: Flight path vector (Source: Boeing).

correction is achieved, then pointed back at the touchdown zone. As shown in Figure 20, the HUD can display a glidepath reference line in order to confirm the desired descent angle, in this case, three degrees. The aircraft in Figure 20 is slightly right of centreline, in order to correct this, the pilot would move the FPV slightly left of the runway centre until re-establishing. If a crosswind were present, the FPV would be offset from the centre of the display, but the technique would be identical (the use of de-clutter mode), is suggested when landing with a significant crosswind). Similarly, the three degree reference line can be repositioned up or down the runway by manoeuvring the FPV above or below it respectively. The use of HUD to fly an accurate visual approach is easily learned once the key concepts are grasped, the required skills are an extension of the already familiar aspects of visual flight.

The FPV also has uses in other phases of flight, in particular, the avoidance of weather. As the FPV predicts the future position of the aircraft with reference to the visual scene, simply pointing the vector above or to the side of a storm cloud will ensure the aircraft remains clear (provided there are no power or configuration changes).

Speed control: The FPV displays information about two axes of velocity, in order to complete the picture, information relating to speed and linear acceleration is required. This is achieved by the additional symbology shown in Figure 21:

1. Flight path acceleration symbol - distance above FPV wing denotes level of positive linear acceleration, below the wing shows deceleration.

2. Speed error tape - displays difference between selected (bugged) speed and indicated speed.

In this case, the aircraft is above selected speed and accelerating. In order to resolve this speed error the pilot would reduce thrust, resulting in the acceleration symbol moving below the FPV wing. Once the error tape reaches zero, thrust would be increased to the extent that the acceleration symbol is level with the FPV wing.

\section{Low visibility operations}

One of the main benefits of the HUD is the ability to operate in lower visibility conditions while maintaining situational awareness and precise aircraft control. While non-HUD aircraft have been approved to conduct autoland approaches in conditions of extremely low visibility, the need to manually control the aircraft on takeoff has been more limiting. The use of HUD results in a minimum takeoff visibility of just 75 meters in the 787 as well as increasing the situational awareness of the pilot flying $(\mathrm{PF})$ during a low visibility autoland.

HUD takeoff: The HUD system meets FAA and ICAO requirements for a takeoff guidance system, allowing low visibility takeoff from a category III runway with as little as 75 meters runway visual range (RVR), this is half the lowest minimum available without the HUD. While Boeing assert that primary guidance should be achieved by visual reference to the runway markings and centreline lighting [1], the steering information provided by the HUD ground-roll guidance cue significantly improves situational awareness. Figure 22 shows the aircraft to the left of centreline in 75 meter RVR conditions. In addition to the familiar localiser display beneath it, the guidance cue is showing a 'drive right' command. The HUD also assists with rotation rate precision and although less of an issue in the 787, HUD pitch-limit information reduces the risk of tail strike in aircraft such as the 737$800 / 900$ which are more prone to this.

HUD landing: Traditional autoland technique has the PF looking out the windscreen and the pilot monitoring (PM) confirming correct operation of the autopilots and monitoring the instruments during the final stages of approach. Using HUD allows the PF to stay 'in the picture' while scanning for visual contact with the runway. Figure 23 highlights the importance of correct contrast setting during this critical phase, if the HUD is set too bright, it may be difficult to make out the runway markings as they come into view.

Rollout and deceleration: Another key feature of the HUD is the 
Citation: Nichol RJ (2015) Airline Head-Up Display Systems: Human Factors Considerations. Int J Econ Manag Sci 4: 248. doi:10.4172/21626359.1000248

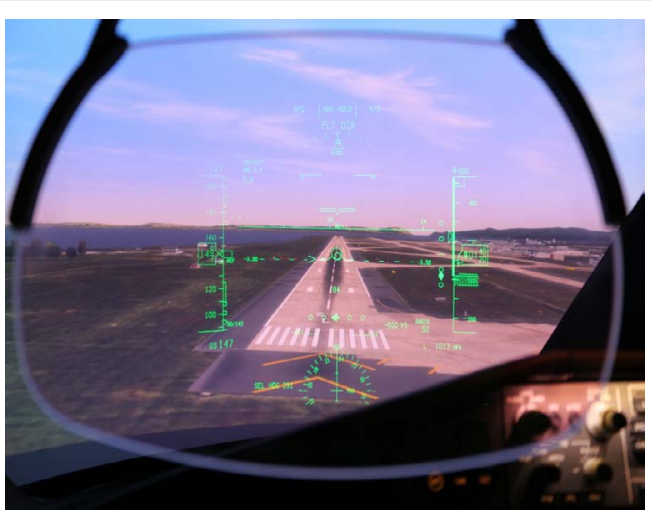

Figure 20: Visual approach using FPV.

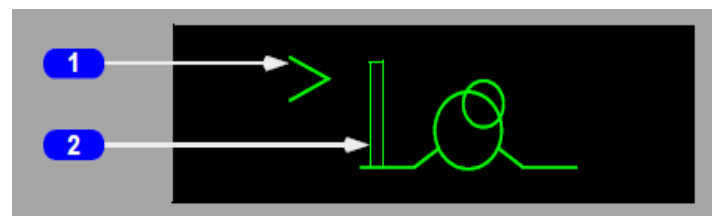

Figure 21: FPV speed symbols (Source: Boeing).

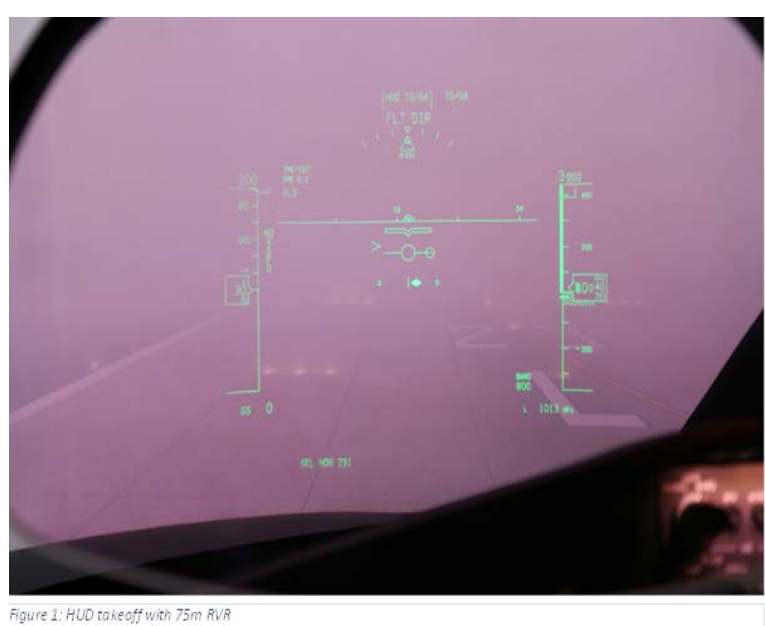

Figure 22: HUD takeoff with $75 \mathrm{~m}$ RVR.

ability to monitor deceleration performance during landing rollout or perhaps more importantly, rejected takeoff. Without HUD it is difficult for the PF to monitor aircraft performance while managing the rollout and they must largely rely on calls from the PM, confirming autobrake function and deceleration. Figure 24 shows the HUD in declutter mode during landing rollout. The scale below the localiser display indicates deceleration rate referenced to autobrake calibration rates, in this case the autobrake three rate is (approximately) being achieved, as selected.

\section{Conclusion and Recommendations}

This report has surveyed the relevant aspects of aviation human factors in an effort to fully appraise the use of head-up display systems in an airline environment. HUD systems are likely to become commonplace in commercial aircraft as their benefits are realised by an increasing number of users. While the HUD is an extremely useful piece of equipment, like many advances in technology, there are aspects of HUD use that pose the potential for difficulty, particularly the attention capture phenomenon. Additionally, the reliance on HUD during safety critical phases of flight such as low visibility takeoff and landing emphasises the need for adequate training and practice. There is more to be learned about the application of HUD technology in an airline environment. The following recommendations are aimed at maximising the usefulness of what is currently understood, and the advancement of operational knowledge surrounding head-up display use:

1. Training: It is essential that crews undertaking initial training on the HUD are made fully aware of its limitations and taught mitigating skills and techniques. While it is likely that training in the technical aspects of the HUD, such as practical use and displayed symbology, would be well covered by standard aircraft type training, focus should also be given to attention capture. It is therefore recommended that crews are given adequate background information surrounding the role of visual attention in HUD use, and taught the importance of using correct contrast ratios and continuing to 'scan' between the HUD symbology and outside world just as they would for a head-down display. The idea of looking past the HUD symbology and focussing on the visual scene should be emphasised as a way to properly include outside cues in the visual scan.

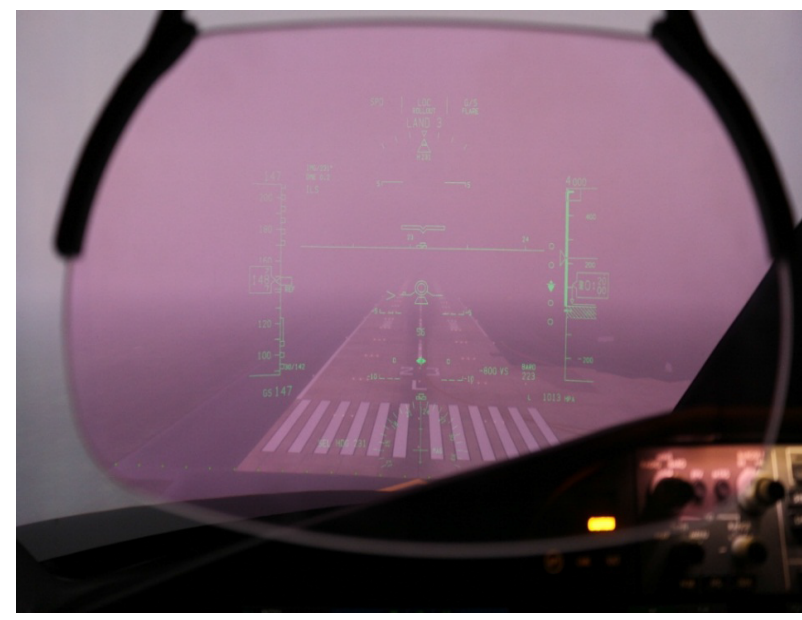

Figure 23: HUD during autopilot landing.

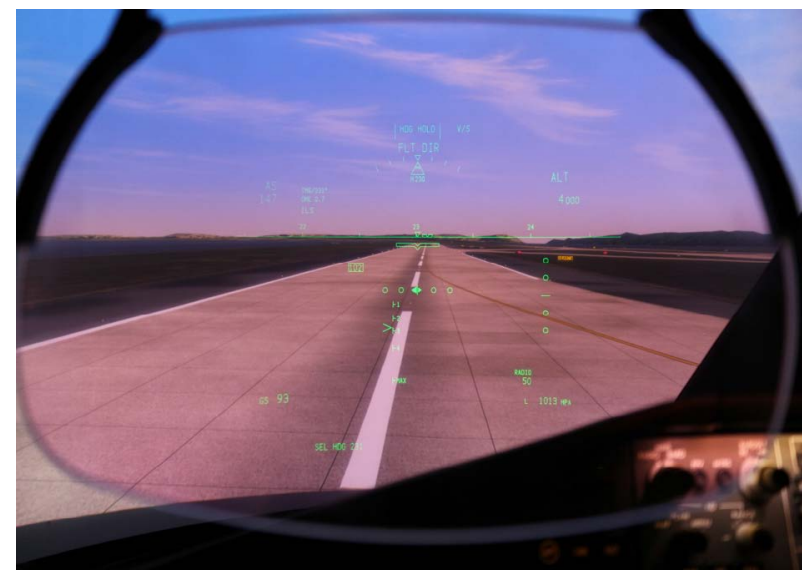

Figure 24: HUD in declutter mode showing rollout deceleration. 
2. Standard operating procedures: Given the importance of experience and habit-forming in flying, it would be wise to consider the implications of HUD use in critical situations such as low visibility takeoff and landing. For example, it would not be appropriate to only use the HUD for takeoff when required to do so by low visibility. It is recommended that the use of HUD is mandatory for both pilots during each takeoff and landing, and that the FMC HUD takeoff mode is used whenever available. This not only ensures sufficient familiarity when using the system in a low visibility environment, but promotes higher levels of general HUD experience amongst crews, likely to be a risk mitigating factor in the day-to-day operation of the aircraft. The HUD takeoff mode also mitigates the risk of misidentifying either the takeoff runway, or centreline lights at night.

3. Data collection: There is a need to gather information about HUD use in an operational (rather than research-based) setting. It would be very useful for an airline to elicit information from crews transitioning to the HUD as they gain experience, and continue to encourage the reporting of events such as the discontinuation of HUD use during turbulence or attention capture by HUD symbology. It is advisable that airlines track sub-occurrence level information with respect to operational areas that are new and/or not well understood, the use of HUD is one such area.

\section{References}

1. Draeger J (2009) Visual ergonomy and flying safety. The importance of recognizing the instruments. Ophthalmologe 106: 370-373.

2. Dragoi V (2012) Ocular motor system. Neuroscience Online: An Electronic Textbook for the Neurosciences. Retrieved 22

3. Travis RC (1948) 38: 395-403.

4. Watson NV, Breedlove S (2012) The mind's machine: Foundations of brain and behavior. Sunderland MA: Sinauer Associates.

5. Firth AY, Davis H, Horwood AM (2013) Binocular visual acuity in intermittent exotropia: Role of accommodative convergence. American Journal of Ophthalmology 155: 776-777

6. Isayama T, Zimmerman AL, Makino CL (2009) The molecular design of visual transduction.

7. Chabre M, Deterre $P$ (1989) Molecular mechanism of visual transduction European Journal of Biochemistry 179: 255-266.

8. Leibowitz HW (1988) The human senses in flight. In E. L. Wiener \& D. C. Nage (Eds.), Human factors in aviation. London, UK: Academic Press

9. Wickens CD, Flach JM (1988) Information Processing In EL Wiener, DC Nage (Eds.), Human factors in aviation. London, UK: Academic Press.

10. Gibb R Gray, Scharff L (2010) Aviation visual perception: Research, misperception and mishaps. Surrey, UK: Ashgate.

11. Sugano D (2005). Cell phone use and motor vehicle collisions: a review of the studies. Honolulu, HI: Legislative Reference Bureau

12. Vecera SP, Cosman JD, Vatterott, DB, Roper ZJJ (2014) The control of visual attention: Toward a unified account. In HR.Brian (Ed.) Psychology of Learning and Motivation, 303-347.

13. Hollingworth A, Maxcey-Richard AM (2013) Selective maintenance in visual working memory does not require sustained visual attention. Journal of Experimental Psychology: Human Perception and Performance 39: 1047-1058.

14. Griffin IC, Nobre AC (2003) Orienting attention to locations in interna representations. Journal of Cognitive Neuroscience 15: 1176-1194

15. Makovski T, Sussman R, Jiang $Y$ (2008) Orienting attention in visual working memory reduces interference from memory probes. Journal of Experimental Psychology: Learning, Memory, and Cognition, 34: 369-380.
16. Matsukura M, Luck SJ, Vecera SP (2007) Attention effects during visual short-term memory maintenance: Protection or prioritization? Perception \& Psychophysics 69: 1422-1434.

17. Vcervers PM, Wickens CD (1998) Conformal flight path symbology for head-up displays: defining the distribution of visual attention in three-dimensional space: University of Illinois at Urbana-Champaign.

18. Curtis MT, Jentsch F, Wise JA (2010) Aviation displays. In E. Salas and D. Maurino (Eds.), Human Factors in Aviation 439-479.

19. Spehar B, Owens C (2012) When do luminance changes capture attention? Perception \& Psychophysics: 674-690.

20. Wyszecki G, Stiles WS (1982) Color science - concepts and methods, quantitative data and formulae.

21. Williams D (2005) Color perception is not in the eye of the beholder: It's in the brain.

22. Arditi, A (2013) Effective color contrast.

23. Jukes M (2004) Aircraft display systems London UK: Professional Engineering Publishing.

24. Suren I (2000) Avionics \& flight management systems for the air transport pilot (3rdedn). South Melbourne: Aviation Theory Centre.

25. Flight crew operations manual (2013) Boeing Aircraft Corporation.

26. Jarrett DN (2005) Cockpit engineering. Burlington VT: Ashgate Publishing.

27. Collinson RPG (2011) Introduction to avionics systems Dordrecht: Springer.

28. Sweetman B (2011) Point and Shoot. Defense Technology International 5: 15-15

29. Crawford J, Neal A (2006) A Review of the Perceptual and Cognitive Issues Associated With the Use of Head-Up Displays in Commercial Aviation. International Journal of Aviation Psychology 16: 1-19.

30. Montgomery T (2014) Visual acuity.

31. Wood RB, Howells PJ (2007) Head-up displays in CR Spitzer (Ed.) Digital avionics handbook Boca Raton, FL: CRC Press.

32. Homan M (2013) The use of optical waveguides in head up display (HUD) applications. Paper presented at the Display Technologies and Applications for Defense, Security, and Avionics VII.

33. Proctor $P$ (1998) Hud for all reasons. Aviation Week \& Space Technology: 13

34. Proctor $P$ (1999) HUDs may help in smoky cockpit. Aviation Week \& Space Technology 150

35. Fadden S, Wickens CD, Ververs PM (2000) Costs and benefits of head-up displays: An attention perspective and a meta analysis.

36. Wickens CD, Alexander AL (2009) Attentional tunneling and task management in synthetic vision displays. The International Journal of Aviation Psychology 19: $182-199$

37. Wickens CD, Helleberg J, Goh J, Xu X Horrey WJ (2001) Pilot task management: Testing an attentional expected value model of visual scanning Savoy, IL: University of Illinois Aviation Research Lab

38. Thomas LC, Wickens CD (2001) Visual displays and cognitive tunneling Frames of reference effects on spatial judgments and change detection.

39. Fischer E, Haines RF, Price TA (1980) Cognitive issues in head-up displays Moffett Field, CA: NASA Ames Research Center.

40. Karar VS, Bajpai YP, Garg H (2012) Study of Attention Capture Aspects with respect to Contrast Ratio for Wide Background Luminance Range in Head-up Displays. Lecture Notes in Engineering and Computer Science: 1328.

41. Larish I, Wickens CD (1991) Divided attention with superimposed and separated imagery: Implications for head-up displays. Savoy IL: University of Illinois Institute of Aviation. 